\title{
Complex Supply Networks Evolving Model: Complex Networks Perspective
}

\author{
Gang Li, Qi Xuan, Zhihuan Song \\ Institute of Industrial Process Control \\ Zhejiang University \\ Hangzhou, China \\ zhsong@iipc.zju.edu.cn
}

\author{
Xiaogang Jin \\ Institute of Artificial Intelligence \\ Zhejiang University \\ Hangzhou, China \\ xiaogangj@cise.zju.edu.cn
}

\begin{abstract}
-supply networks modeling has gained tremendous attention in the field of supply chain during last decades. However, most existent literatures in supply networks do not pay much attention to the topological characteristics, which, as revealed by Complex Networks, have close relations with their behaviors in various complex systems. This paper introduces a novel topological structure evolving model of complex supply networks from the perspective of Complex Networks. After investigating supply networks typical structural characteristics and formation mechanisms, Complex Supply Networks Evolving Model (CSNEM) are proposed to mimic these structural characteristics. Then, three statistical properties are investigated. To validate CSNEM, three real supply chain networks are introduced. The results indicate that CSNEM is consistent with the real-world supply networks. Further the relations between these properties and supply networks macro behaviors i.e. rapid response capability and robustness are discussed. This work will provide managers and researches a beneficial insight on macroscopic structures characteristics as well as a helpful understanding on the relations between macroscopic structures and global behaviors in supply networks.
\end{abstract}

Keywords- Supply networks; Global topological structures; Complex networks; Evolving model

\section{INTRODUCTION}

Supply networks involve complex interactions among suppliers, manufacturers, distributors, third-party logistics providers, retailers, and customers. The numerous interactions between entities as well as the characteristics of nonlinearity, dynamics etc. in supply networks make it challenging to analyze and predict their responses over time. So far, considerable endeavors have been made to construct models and predict their performance ${ }^{[1,2]}$. The conventional models of supply chains in the literatures mainly focus on the issue from the three levels: strategic level, which includes location/allocation decisions, demand planning, distribution channel planning etc; tactical level, which covers inventory control, production distribution coordination, order/freight consolidation etc. and operational level, vehicle routing/scheduling, workforce scheduling, record keeping, and packaging belong to this level ${ }^{[2]}$. These previous researches provide a beneficial insight on supply chain and they address problems mainly from the microscopic view i.e., focusing on either the focal entity or relations between two entities in the supply networks ${ }^{[3]}$.

Nowadays, the increase of customer expectation, the enlargement of outsourcing as well as the globalization of trade have led to supply networks much complicated. Most researchers come to realize that supply networks are representative complex systems ${ }^{[4]}$. In this case, some modern modeling approaches are introduced to model these complex supply networks ${ }^{[5-7]}$. The most important ones are multi-agent systems (MAS) and complex adaptive systems (CAS). MAS addresses the various entities in supply chain networks as heterogeneous agents and agents interact with other agents via communication protocol languages ${ }^{[7]}$. While CAS takes supply networks as living systems and the networks co-evolve with their dynamic environments ${ }^{[5,6]}$. Both of these approaches take supply networks as self-organizing systems and try to explain how supply chain networks structures emerge.

Recently, as an effective approach to complex systems, Complex Networks have been obtained ongoing attention and applied to various of fields, such as social networks, technological networks and information networks et al.. Complex Networks try to investigate the mechanisms that determine the topological structures of complex systems and explore the relations between these structural characteristics and their behaviors in complex systems. Recent results reveal that there are close relations between them ${ }^{[8-11]}$. Supply networks, as typical complex systems, can also be studied from the perspective of Complex Networks ${ }^{[12-16]}$. Far from traditional methods, Complex Networks, stressing the systemic complexity of systems, will facilitate the design of policies that reduces the conflict between companies' interests and global efficiency as well as reduce the risks of global failure to make the networks more robust ${ }^{[16]}$. With Complex Networks approach, we can model supply networks structures from the macroscopic view, analyze the relations between their macrostructures and behaviors, and further predict their behavioral tendencies. These will provide managers and researches a beneficial systemic insight on their performance since supply networks are integrated systems, high local performance do not definitely lead to high global performance. Thadakamalla et al. firstly studied supply networks modeling from the perspective of Complex Networks ${ }^{[12]}$. The authors focused on the feature of survivability in military supply networks, and analyzed the relations between the survivability and their topological structure, further proposed a model to meet the characteristic. Some other researchers also investigated supply networks topological characteristics from the perspective of Complex Networks ${ }^{[13-15]}$. These pioneer works provide beneficial endeavors in supply networks

This paper is supported by the National Natural Science Foundation of China (Grant No.60872057) 
researches. While the problems about the structural characteristics and formation mechanisms in real-world supply networks have not gotten sufficient attention.

In this paper, after investigating supply networks structural characteristics and formation mechanisms, we propose Complex Supply Networks Evolving Model (CSNEM) from the perspective of Complex Networks to mimic these characteristics. Then we analyze three fundamental statistical properties in CSNEM which include average path length $\boldsymbol{L}$, clustering coefficient $\boldsymbol{C}$ and degree distribution $\boldsymbol{P}(\boldsymbol{k})$. The simulation and analysis reveal that in supply networks, the value of average path length is comparatively small and dose not increase correspondingly as networks size grows, known as the small-world property; the clustering coefficients is proximate to zero; and the degree distribution follows powerlaw distribution, i.e. Scale-Free network. These results are validated by the empirical study. In addition, we discuss the relations between these statistical properties and their behaviors in supply networks, i.e. rapid response capability and robustness.

The remainder of this paper is organized as follows. In Section 2 , supply networks structural characteristics and the formation mechanisms are introduced. Next, in Section 3, Complex Supply Networks Evolving Model (CSNEM) based on Complex Networks is proposed. Analytical and simulation results, a case study as well as some discussions are given in Section 4. Finally, conclusions and further research directions are discussed in Section 5.

\section{SUPPLY NETWORKS STRUCTURAL CHARACTERISTICS AND FORMATION MECHANISMS}

Supply networks are typical complex systems that consist of a variety of suppliers, manufacturers, distributors, retailers and costumers. Their structures can be described in two dimensions, as shown in Fig.1. : the horizontal and vertical dimensions. The horizontal dimension refers to the number of tiers across the supply networks, while the vertical dimension denotes the number of entities in each tier ${ }^{[17]}$. Supply networks' structures may take on long-wide or short-narrow characteristics as the numbers of horizontal dimensions or vertical ones are different in various supply networks.

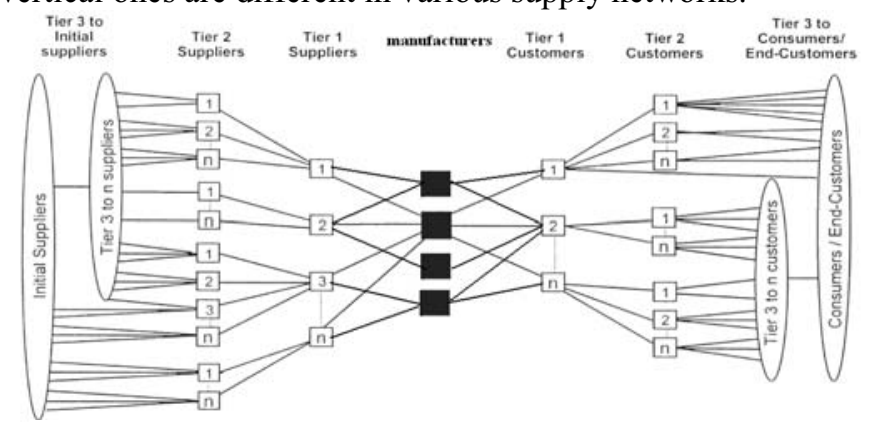

Fig.1. Supply network structure (Source adapted from [17])

\section{A. Supply networks structural characteristics}

Supply networks display some structural characteristics which include complexity, uncertainty, hierarchy and dynamics etc. Here, we mainly introduce two of main structural characteristics, i.e. hierarchy and dynamics. These two characteristics are common in most of supply networks. We mainly focus on these two characteristics in CSNEM.

Hierarchy: From topological perspective, supply networks structures take on hierarchical characteristic. This is distinguished in industrial supply networks, e.g. automotive industry ${ }^{[18]}$. There are multiple tiers in hierarchical supply networks and each entity belongs to a certain tier. Different tiers perform different functions in the whole supply network. The relations (here, we refer to demand-supply relation) only occur between entities and their partners in previous or following tier, and do not occur in the entities of the same tier. Dynamics: Dynamics is another characteristic. Supply networks are not stationary. They are dynamically evolving over time. From topological perspective, the dynamics are reflected on both alteration in entities number and interacting relations ${ }^{[4]}$. The number of entities in supply networks is changing as some entities may join or exit from the networks due to various reasons. In addition, relations between entities are changeable: some relations may be strengthened and some may be weakened.

\section{B. Supply networks formation mechanisms}

As mentioned before, supply networks display some topological characteristics. The problems about how these structures emerge and which factors determine their formation now attract many researchers' interests ${ }^{[5-7]}$. Here, we investigate these formation mechanisms from the perspective of Complex Networks.

Generally, new supply networks are formed from primitive entities. That is to say, the initial supply networks are composed of few entities. Then, some exterior entities may join the networks over time. These new entities and the existent entities connect each other through mutual selection. Obviously, there are also some entities exiting from the networks, but historically, prosperous supply networks are gradually growing in size. When some exterior entities intend to join the supply networks, which entities will they connect to? Apparently, these connections have preference, and involve partner selection criteria that have been much studied in the fields of supply chain. Whereas partner selection criteria are so complicated and consist of various factors such as cost, quality, and lead time etc. It is hard to take all these factors into a single supply networks model. In order to conquer this difficulty, some researchers adopted the concept of "fitness" to depict the capacity of entities in supply networks $[5,6]$. "Fitness" refers to the ability of an entity to live and thrive in its market environment ${ }^{[5,6]}$. Choi et al. pointed out that an entity should ensure the acceptable cost, delivery, and quality of parts and raw materials purchased in order to increase its fitness ${ }^{[5]}$. Therefore, the "fitness" synthesizes various factors to determine partners' selection, and it can be regarded as entities' attracting ability to its partners. In CSNEM, the fitness strength is viewed as the only factor that determines partners' selection criterion. From the perspective of Complex Networks, we can attribute these mechanisms to two factors: growth and preference connection, which are considered to be 
main formation mechanisms in a variety of complex systems $[8]$

\section{COMPLEX SUPPLY NETWORKS EVOLVING MODEL}

\section{A. Model assumption}

To mimic supply networks characteristics mentioned before, we stipulate CSNEM satisfies the following assumptions which include I) nodes and connections attributes ,II) initial structures and modularity, III) evolving mechanisms .

Nodes and connections attributes:, Nodes in CSNEM represent entities, connections between nodes refer to demandsupply relations in supply networks. Nodes have two attributes: fitness strength and connection degree. The connection degree $\boldsymbol{D}_{\boldsymbol{i}}$ is defined as the sum of a node's connections. A node's fitness strength $\boldsymbol{S}_{\boldsymbol{i}}$ indicates an attracting capacity of node $\boldsymbol{i}$. Its value is equal to the sum of the node's connection weights. As for a connection, it has one attribute, connection weight $\boldsymbol{w}_{i j}$ which denotes intensity of demand-supply relation between entities. Its initial value is fixed at $\boldsymbol{w}_{\boldsymbol{0}}$ uniformly. The value of weight $\boldsymbol{w}_{i j}$ in CSNEM is dynamically changed over time.

Initial structures and modularity: As mentioned before, supply networks are usually formed from primitive entities. Here, we postulate that initial supply networks are three entities which compose the simplest demand-supply relation. That is to say, there are a resource provider, a producer and a customer in the initial structure. Then some exterior entities may join supply networks over time, which results in gradual growth in the networks size. Generally speaking, these exterior entities can not survive in the markets environment independently in most cases. They always have upstream and downstream partners. In this case, a fundamental module is introduced that is composed of three nodes forming a chain structure. These three nodes are Supplier $(\boldsymbol{S})$, Producer $(\boldsymbol{P})$, and Customer $(\boldsymbol{C})$. So in CSNEM, exterior entities as a module join the supply networks.

Evolving mechanisms: When the exterior entities intend to join the supply networks, they must select some existent entities as their partners, which involve partner's selection criteria. Fitness is considered as the only criterion as discussed in Section 1.2. Nodes with high fitness strength have the priority to be connected. In order to guarantee the networks' hierarchical characteristic, a new connection only occurs between node $\boldsymbol{S}$ in the new module and one of existent nodes. Once the connection occurs, the selected node's fitness strength will be altered accordingly. The fitness strength adjustment involves a tunable parameter $\boldsymbol{\delta}$, which demonstrates the impact on selected nodes' fitness strength: the larger is value of $\boldsymbol{\delta}$, the greater increase on the value of fitness strength. Details of the adjusting rule will be introduced in Section 2.2.

\section{B. The evolving model}

Under those assumptions, we propose CSNEM adapted from related evolving models ${ }^{[19-21]}$. CSNEM algorithm is described as follows:
Step 1: Initialization: In initial state, CSNEM generates primitive supply network composed of three initial entity nodes, $\boldsymbol{S}_{\boldsymbol{1}}, \boldsymbol{P}_{\boldsymbol{1}}$ and $\boldsymbol{C}_{\boldsymbol{1}}$. Connection degree and fitness strength are illustrated in Figure 2.

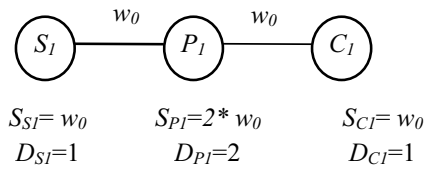

Fig.2. Initial state of CSNEM

$\boldsymbol{S}_{\boldsymbol{i}}$ is the fitness strength of node $\boldsymbol{i}, \boldsymbol{D}_{\boldsymbol{i}}$ is the degree of node $\boldsymbol{i}$. $\boldsymbol{S}_{\boldsymbol{i}}$ defined in Eq.(1), where the sum runs over the set $V(i)$ of neighbors of node $i$.

$$
s_{i}=\sum_{j \in V(i)} w_{i j}
$$

Step 2: Evolving rules: At each time step, according to the assumption II and III, CSNEM generates a module consisting of three nodes .It is similar to the initial supply network. The supplier node in the new module selects only one from existent nodes based on fitness preference defined in Eq.(2) ,where $\sum_{l} s_{l}$ refers to the whole network's fitness strength. The selected node gets a new connection.

$$
\prod_{i \rightarrow l}=\frac{\mathrm{s}_{i}}{\sum_{l} \mathrm{~s}_{l}}
$$

Step 3: Connection's weight adjusting: When node $\boldsymbol{l}$ gets a new connection from node $\boldsymbol{i}$, the existent connections weights of node $\boldsymbol{l}$ are dynamically adjusted. The adjustment rule involves the tunable parameter $\boldsymbol{\delta}$. A larger $\boldsymbol{\delta}$ in value means the more impact on $\boldsymbol{w}_{\boldsymbol{l}}$. Figure 3 demonstrates the detailed illustration about this adjusting rule.

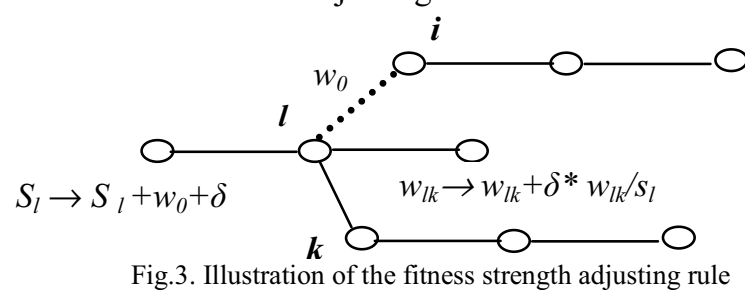

Step 4: Growth: Iterating step 2 and step 3, CSNEM will generate a network with defined size. Figure 4 is the network topological structures with 15 nodes.

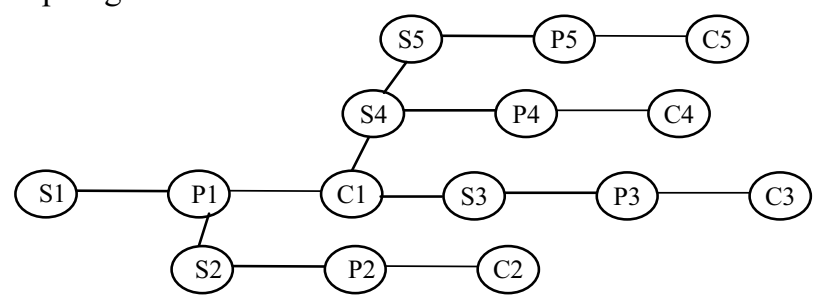

Fig.4. Supply networks topological structure with 15 nodes

\section{MODEL ANALYSIS AND DISCUSSIONS}

In Complex Networks, there are three fundamental statistical properties. They are average path lengths $\boldsymbol{L}$, clustering 
coefficient $\boldsymbol{C}$ and connection degree distribution $\boldsymbol{P}(\boldsymbol{k})$. These properties are defined as follows:

Average path lengths $\boldsymbol{L}$ : the distance $\boldsymbol{d}$ refers to the least number of connections between node pairs. The average path length $\boldsymbol{L}$ is the mean of all $\boldsymbol{d}$ in the network. The quantity of $\boldsymbol{L}$ can effectively indicate the transmission performance of whole networks. In most of real networks, despite of their large size, there is a relatively average path length. This feature is known as the small-world property.

Clustering coefficient $\boldsymbol{C}$ : The clustering coefficient $\boldsymbol{C}$ is the probability that two nearest neighbors of a node are also nearest neighbors of one another. The clustering coefficient of the network reflects the "cliquishness" of the mean closest neighborhood of the network.

Degree distribution $\boldsymbol{P}(\boldsymbol{k})$ : A node's degree $\boldsymbol{k}$ is the total number of its connections. The degree of node $\boldsymbol{i}, \boldsymbol{k}_{\boldsymbol{i}}$ indicates its importance in the network. Degree distribution in a network can be described by the function $\boldsymbol{P}(\boldsymbol{k})$ which is the probability of random selected nodes with degree equal to $\boldsymbol{k}$, and it is also a fundamental statistical characteristic. If a network's degree distribution follows power-law distribution, it is called Scale-Free network.

\section{A. Model analysis}

Average path lengths $L: U p$ to now there is not an effective approach to solving the value of $\boldsymbol{L}$ exactly except for some specific models. However, the changing tendency of $\boldsymbol{L}$ in CSNEM can be obtained by simulation results of CSNEM (showed in Figure 5 and Figure 6), As shown in Figure 5, the average path length $\boldsymbol{L}$ does not increase correspondingly as networks grows in size. In addition, the adjusting parameter $\boldsymbol{\delta}$ has significant impact on $\boldsymbol{L}$, as demonstrated in Figure 6: $\boldsymbol{L}$ will gradually decrease as the value of $\boldsymbol{\delta}$ increases.

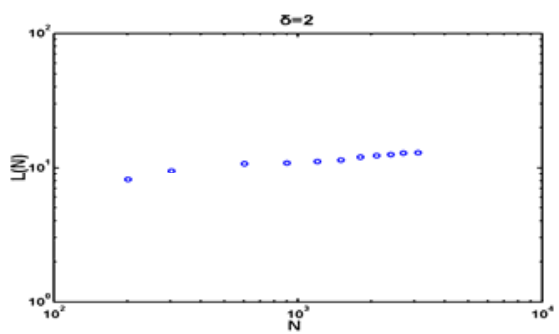

Fig.5.Average path lengths with $N=300$

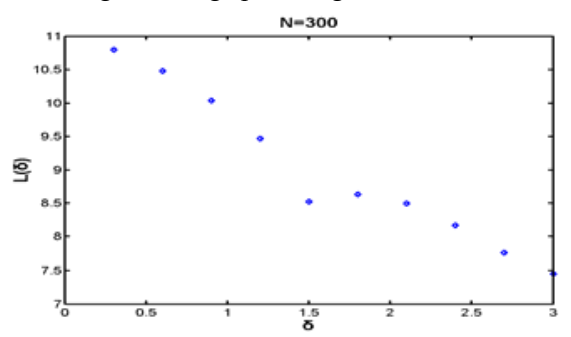

Fig.6.Average path lengths with $\delta=2$

Clustering coefficient $\boldsymbol{C}$ : In CSNEM, a connection occurs only between a supplier node and one of the existent nodes at each time step. Therefore, the networks clustering coefficient $\boldsymbol{C}$ is zero. This is consistent with most cases in real-world supply networks, that is, entities in the same tiers normally have no demand-supplier relations.

Degree distribution $\boldsymbol{P}(\boldsymbol{k})$ : In order to calculate simplicity, we let $\boldsymbol{w}_{\boldsymbol{0}}$ equal to 1 . Each time step, CSNEM adds a module consisting of three nodes. As the new module adds into the network, this will result in the fitness strength of whole network enhanced by $5+2 * \delta$. Without considering the initial fitness strength, as time evolves, the fitness strength of whole network can be equal to $(5+2 * \delta) *$ t. Thus we can get the changing rate of fitness strength according to preference attachment probability.

$$
\frac{d s_{i}}{d t}=\frac{s_{i}}{\sum_{j} s_{j}}(1+\delta)+\sum_{j \in V(i)} \frac{s_{i}}{\sum_{j} s_{j}} \delta \frac{w_{i j}}{s_{j}}
$$

The first item of Eq. (3) indicates node $i$ selection probability and the second item denotes the set of adjacent node of $i$ selection probability. Both situations will lead to variation of the fitness strength $S_{i}$ of node $i$.

$$
\frac{d s_{i}}{d t}=\frac{1+2 \delta}{5+2 \delta} \frac{s_{i}}{t}
$$

In addition, the degree of node $\boldsymbol{i}$ can be expressed by $\boldsymbol{k}_{\boldsymbol{i}}$, the changing rate of $\boldsymbol{k}_{\boldsymbol{i}}$ is proportion to $\boldsymbol{S}_{\boldsymbol{i}}$, so the changing rate of $\boldsymbol{k}_{\boldsymbol{i}}$ can be expressed as Eq. (5)

$$
\frac{d k_{i}}{d t}=\frac{s_{i}}{\sum_{j} s_{j}}=\frac{s_{i}(t)}{(5+2 \delta) t}
$$

Eq. (6) is the solution from Eq. (5),

$$
k_{i}=\frac{(t / i)^{\frac{1+2 \delta}{5+2 \delta}}}{1+2 \delta}=\frac{s_{i}(t)}{1+2 \delta}
$$

According to continuous field theory,

$$
P(k, t)=\frac{1}{3+3 t} \int_{b}^{t} \delta\left[k-k_{i}(t)\right] d i
$$

Solving Eq. (7); we obtain the degree distribution,

$$
P(k) \sim k^{-\gamma}
$$

Where $\gamma=\frac{4 \delta+6}{2 \delta+1}=2+\frac{4}{2 \delta+1}$

Similarly, we can also get the fitness distribution,

$$
P(s) \sim s^{-\gamma}
$$

From above analytical results, we can conclude that the networks generated by CSNEM follow power-law distribution, and the fitness strength also satisfies power-law distribution. The exponent of power-law is related to adjusting parameter $\boldsymbol{\delta}$ (as showed in Eq.(9)). Figure 7 and Figure 8 demonstrate numerical simulation results of fitness strength and degree distribution. These implicate that in supply networks, there are few entities which own more connections and more fitness capacity than the most of other entities own. From the real-world American auto industrial supply networks investigated by Choi et al ${ }^{[18]}$, we can clearly see that there definitely exist few entities that have more connections with other entities in the same supply networks than the most 
remainder have. This result is also agreement with some prior researches. For example, Sun and $\mathrm{Wu}$ analyzed the supply networks where data were obtained from the Internet, and concluded that supply networks follow power-law distribution [15]; another example is that Kühnert et al. pointed out that urban supply networks also satisfy power-law distribution ${ }^{[13]}$.

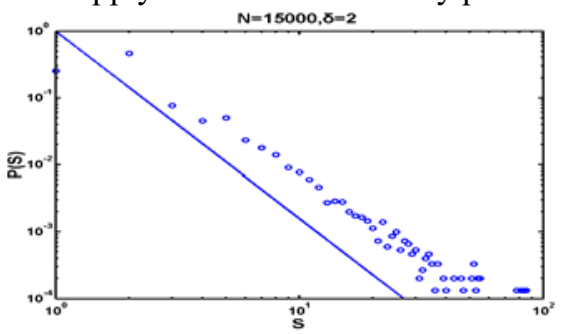

Fig.7.The fitness distribution with $N=15000, \delta=2$

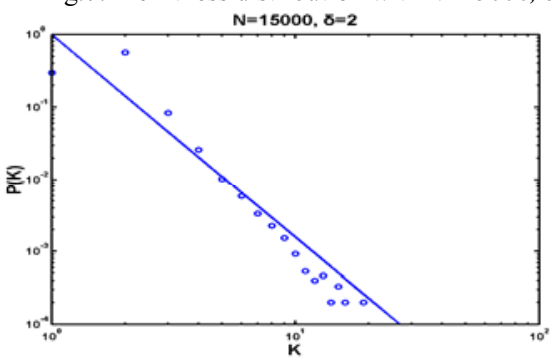

Fig.8. The degree distribution with $N=15000, \delta=2$

From simulation and theoretical analysis, we can get the conclusion that the value of average path length in a supply network is comparatively small and does not increase correspondingly as networks size grows, i.e. the small-world property; its degree distribution follows power-law distribution, i.e. Scale-Free network, and its clustering coefficients is proximate to zero.

\section{B. Case study}

Source of the data here is from precursors' research since data of real-world supply networks are challenging to gather. Choi and Hong undertook an empirical study on three automatic industrial supply networks in USA: Honda Accord, Acura CL/TL, and DaimlerChrysler (DCX) Grand Cherokee, and presented the topological graphs of these three supply networks in their paper ${ }^{[18]}$. The statistical properties of these supply networks are calculated according to their definitions and are tabulated below (see Table I). As shown in Table I, clustering coefficient $\boldsymbol{C}$ is much small and approximate to zero, and the value of average path length $\boldsymbol{L}$ is also small. Table I also shows the values of average degree $\langle\boldsymbol{k}>$ in these supply networks. As for degree distribution $\boldsymbol{P}(\boldsymbol{k})$, their degree distributions are shown in Figure 9. Due to the limited size of these supply networks, it is not obvious that $\boldsymbol{P}(\boldsymbol{k})$ follows strictly power-law property. However it still shows that there are some entities which own much large value of degree than the others, which is to some extent agreement with CSNEM.

Table I: Topological characteristics in Honda Accord, Acura CL / TL and DaimlerChrysler

\begin{tabular}{c|c|c|c}
\hline Supply Networks & $C$ & $L$ & $<k>$ \\
\hline Honda Accord(51 nodes) & 0.0232 & 3.9565 & 2.4706 \\
\hline Acura CL / TL (77 nodes) & 0.0049 & 3.6337 & 2.3766 \\
\hline DaimlerChrysler (42 nodes) & 0 & 3.3856 & 2 \\
\hline
\end{tabular}

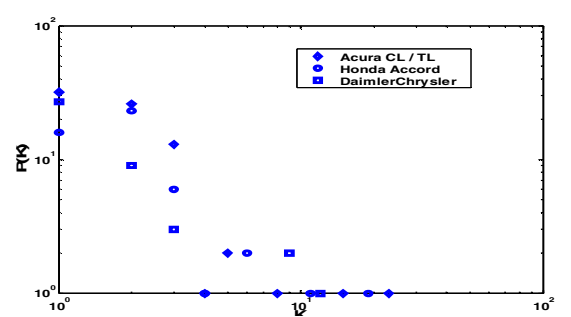

Fig.9.The degree distribution in three real supply networks

\section{Discussions}

Complex Networks reveal that there are close relations between their topological structures and behaviors in many complex systems. This paper provides discussion on these relations in supply networks concentrating on two main characteristics, namely, rapid response capability and robustness.

Rapid response capability: With growth of customers demand, rapid response capability has been increasing importance for supply networks. Supply networks with rapid response capability will be agile to the variations in customer demands and markets, and get predominance on competitive market environments ${ }^{[22]}$. A better topological structure can improve supply networks responsiveness which will enable logistics to flow quickly through few inter-nodes from the initial entities to their destinations ${ }^{[23]}$. This networks transmission performance is related to average path length $\boldsymbol{L}$. If the value of $\boldsymbol{L}$ is smaller, the transmission performance will be more efficient. Thus it will result in the improvement in rapid response capability.

From the topological perspective, in order to speed up the communication among any two nodes, it is imperative for supply networks with comparatively small value of $\boldsymbol{L}$.CSNEM and empirical study demonstrate that the value of average path length in supply networks is small and does not have a greater increase when supply networks scale grows. In other words, an entity can take only a few hops from itself to another although networks size is much larger. In addition, it is shown in Figure 6 that the parameter $\boldsymbol{\delta}$ has great influence on the value of average path length. The average path length can apparently decrease with increase of $\boldsymbol{\delta}$. While so far, it is difficult to assess the response capability adopting quantitative methods. It may be reasonable to evaluate supply networks response capability by calculating the value of average path length in supply networks.

Robustness: Robustness is vital to supply networks in these competitive markets with lots of uncertain factors. Only in robust supply networks, entities can process efficiently and satisfy the customers' needs and finally get profits ${ }^{[24]}$. Therefore, the researches on the robustness of supply networks have obtained tremendous attention in supply chain management.

Robustness is also one of the research topics in Complex Networks, which explores the connectivity of networks through random and target deleting parts of nodes in networks. Albert et al. revealed that networks with power-law distribution can effectively repel random attack, but expose vulnerability to target attack ${ }^{[25]}$. The theoretical analysis and 
numerical simulation demonstrate that the networks generated by CSNEM follow power-law distribution in the degree and fitness. They are Scale-Free networks, which will be instructive to the robustness research in supply networks. The results in this paper may provide beneficial insights in protecting supply networks. It is suggested that managers should pay more attention to protect few nodes with high connections (networks "hub" in Complex Networks). While some low linked nodes' failures may have little impact on whole supply networks performance.

\section{CONCLUSION AND FUTURE WORK}

In this paper, we explore the structural characteristics and formation mechanisms in supply networks, and propose CSNEM from the perspective of Complex Networks. Three fundamental statistical properties in CSNEM are analyzed which include average path length $\boldsymbol{L}$, clustering coefficient $\boldsymbol{C}$ and degree distribution $\boldsymbol{P}(\boldsymbol{k})$. The simulation and analysis reveal that the value of average path length $\boldsymbol{L}$ is comparatively small and dose not increase correspondingly as networks size grows, which is known as the small-world property; the degree distribution $\boldsymbol{P}(\boldsymbol{k})$ follows power-law distribution; and the clustering coefficients is proximate to zero. These results are validated by the empirical study. In addition, we discuss the relations between these properties and their behaviors in supply networks, i.e. response ability and robustness. This work may contribute some beneficial insights on supply networks researches. However, as Schweitzer et al. pointed out that supply networks are typical economic networks and difficult to predict and control due to dynamic interaction of a large number of different entities ${ }^{[16]}$. There are still more subsequent researches to be carried out. In brief, three directions are suggested to be further explored.

- The evolving mechanisms should be further explored. In CSNEM, for the purpose of simplicity, the networks growth mechanism is the only consideration.

- The relations between behaviors and structural statistical characteristics in supply networks should also be further investigated. The exact relations between structural characteristics and behaviors (e.g. response capability, robustness and so on), are also worthy of investigating.

- As mentioned above, there are close relations between structural characteristics and behaviors. The realistically significant question is whether it is practical to adopt some measures to change structure to avoid some unfavorable behaviors in supply networks. The answers to this question should be further explored.

\section{REFERENCES}

[1] B. M. Beamon, "Supply chain design and analysis: models and methods," International Journal of Production Economics, vol.55, pp. 281-294, April 1998.
[2] H. Min, G. Zhou, "Supply chain modeling: past, present and future," Computers and Industrial Engineering, vol. 43, pp. 231-249, July 2002.

[3] F. T. S. Chan, and H. K. Chan, "The future trend on system-wide modeling in supply chain studies," International Journal of Advanced Manufacturer Technology, vol.25, pp. 820-832. August 2005.

[4] C. C. Bozarth, D. P. Warsing, and B. B. Flynn, "The impact of supply chain complexity on manufacturing plant performance," Journal of Operations Management, vol.27, pp.78-93, August 2008.

[5] T. Y. Choi, K. J. Dooley, and M. Rungtusanatham, "Supply networks and complex adaptive systems: control versus emergence," Journal of Operations Management, vol.19, pp.351-366, May 2001.

[6] S. D. Pathak, D. M. Dilts, and G. Biswas, "On the evolutionary dynamics of supply network topologies," IEEE Transactions on Engineering Management, vol.54, pp.662-672, November 2007

[7]J.H.Lee, and C.O.Kim, "Multi-agent systems applications in manufacturing systems and supply chain management: a review paper," International Journal of Production Research, Vol. 46, pp.233-265, January 2008

[8] R. Albert, and A. L. Barabási, "Statistical mechanics of complex networks," Reviews of Modern Physics, vol.74, pp.47-97, January 2002.

[9] S. N. Dorogovtsev, and J. F. F. Mendes, "Evolution of networks," Advances in Physics, vol.51, pp.1079-1187, March 2002

[10] M.E.J. Newman,"The structure and function of complex networks," Siam Review, vol.45, pp.167-256, March 2003.

[11] S. H. Strogatz, "Exploring complex networks," Nature, vol.410, pp.268276, March 2001.

[12] H. P. Thadakamalla, U. N. Raghavan, S. Kumara, R. Albert, "Survivability of multiagent-based supply networks: a topological perspective," IEEE Intelligent Systems, vol.19, pp.24-31, October 2004.

[13] C. Kühnert, D. Helbing, and G. B. West, "Scaling laws in urban supply networks," Physica A, vol.363, pp.96-103, February, February 2006.

[14] Q. Xuan, Y. Li, T. J. Wu, "A local-world network model based on internode correlation degree," Physica A, vol.378, pp.561-572, November 2007.

[15] H. Sun, and J. Wu, "Scale-free characteristics of supply chain distribution networks," Modern Physics Letters B, vol.19, pp.19841-848, February 2005.

[16] F.Schweitzer, G. Fagiolo, D.Sornette, F. V.Redondo, A. Vespignani, and D.R.White, "Economic Networks: The New Challenges," SCIENCE, vol.325, pp.422-425, July 2009.

[17] D. M. Lambert, M. C. Cooper, and J. D. Pagh, "Supply chain management: implementation issues and research opportunities," International Journal of Logistics Management, vol.9, pp.1-19, November1998.

[18] T. Y. Choi, and Y. Hong, "Unveiling the structure of supply networks: case studies in Honda,Acura, and DaimlerChrysler," Journal of Operations Management, vol.20, pp.469-493,September 2002.

[19] S. N. Dorogovtsev, A. V. Goltsev, and J. F. F. Mendes, "Pseudofractal scale-free web,” Physical review E, vol.65, 066122, January 2002.

[20] E.Ravasz, and A. L.Barabási, "Hierarchical organization in complex networks,” Physical Review E, vol.67, 02611, February 2003.

[21] A. Barrat, M. Barthelemy, and A. Vespignani, "Weighted evolving networks: coupling topology and weight dynamics," Physical Review Letters, vol.92, 228701, June 2004.

[22] C. Y. Wong, J. S. Arlbjørn, and H. H. Hvolby, “Assessing responsiveness of a volatile and seasonal supply chain: a case study," International Journal of Production Economics, vol.104, pp.709-721, February 2006.

[23] A. Gunasekaran, K. H. Lai, and T. C. E. Cheng, "Responsive supply chain: A competitive strategy in a networked economy," Omega-International Journal of Management Science, vol.36, pp.549-564, December 2008.

[24]E.V.Guilherme, and L.Reynaldo, "Understanding supply chain robustness," Proceedings of the IEEE International Conference on Service Operations, Logistics and Informatics, Chicago, USA, pp.157 -162, July 2009.

[25] R.Albert, H.Jeong, and A.L.Barabási, "Attack and error tolerance in complex networks," Nature, vol.406, pp.387-482, July 2000 